\title{
The Effectiveness and Safety of Prostaglandin E2 Pessary versus Intravaginal Gel for Induction of Labor
}

\author{
Salman Al Shahed", Asma Al Shammari, Majd Ghawji, Yasser Habash \\ Department of Obstetrics \& Gynecology, Security Forces Hospital Program, Riyadh, Saudi Arabia
}

\author{
DOI: $10.36348 /$ sijog.2020.v03i02.002
}

| Received: 21.01 .2020 | Accepted: 01.02.2020 | Published: 14.02.2020

*Corresponding author: Salman Al Shahed

\section{Abstract}

Background: The aim of induction of labor is to initiate labor when maternal and fetal conditions necessitate delivery before the onset of spontaneous contractions. The success of this obstetric practice is highly dependent upon the condition of the cervix. Cervical ripening is a complex process that results in physical softening and distensibility of the cervix, ultimately leading to partial cervical effacement and dilatation. Objective: The purpose of the current study was to compare the effectiveness and safety of the pessary to intravaginal dinoprostone gel for induction of labor. Results: A total of 200 women were included in the study, of whom100 received dinoprostone pessary and 100 dinoprostone intravaginal gel. Tachysystole was diagnosed by the research team in 33 women, $(4.5 \%)$ of those received the pessary and $(2.4 \%)$ of those received gel (Table 4$)$. In Caesarean section for suspected fetal compromise associated with uterine Tachysystole was performed in seven women in the pessary group and one in the gel group. There was no correlation between the initial Bishop score and the occurrence of uterine Tachysystole. $(4.1 \%)$ women in the pessary group had a postpartum haemorrhage with estimated volume of $\geq 1000 \mathrm{~mL}$ compared to $(2.2 \%)$ women in the gel group $(P=0.08)$. Conclusion: In this retrospective study, use of dinoprostone vaginal pessaries for induction of labour resulted in no advantage compared with dinoprostone intravaginal gel and clinically significant tachysystole appeared more common.

Keywords: Pregnancy, Induction of Labor, Nulliparous, Multiparous, Tachysystole.

Copyright @ 2020: This is an open-access article distributed under the terms of the Creative Commons Attribution license which permits unrestricted use, distribution, and reproduction in any medium for non-commercial use (NonCommercial, or CC-BY-NC) provided the original author and source are credited.

\section{INTRODUCTION}

The aim of induction of labor is to initiate labor when maternal and fetal conditions necessitate delivery before the onset of spontaneous contractions [1]. The success of this obstetric practice is highly dependent upon the condition of the cervix, and it is well known that an unfavorable cervix is associated with failure of induction, operative vaginal delivery and cesarean section [1]. Prostaglandins have a central role in the physiological events of cervical ripening and parturition, and have been widely used for induction of labor [2]. These can be administered orally, vaginally, intracervically, endovenously and by extra-amniotic or intra-amniotic routes. Dinoprostone is one of the synthetic prostaglandins most commonly used to achieve cervical ripening and labor induction, and can be administered as tablets, suppositories, gel (vaginal and intracervical) or as a controlled-release intravaginal pessary [3].
Epidemiological studies have shown that, after 41 weeks, the rate of fetal, maternal and neonatal complications increases [2]. Therefore, the management of post term pregnancies remains one of the most common obstetric problems in practice. In order to prevent post- term and associated complications, routine induction before 42 weeks has been proposed [3].

The ideal method to induce labor should be safe, painless, inexpensive, comfortable and effective. However, such a perfect method does not currently exist [3].

Most of the available methods of labor induction try to mimic the physiological sequence of cervical effacement and dilation followed by uterine contraction, but the majority achieves only part of the natural progression to labor and delivery. 
Cervical ripening and the administration of oxytocin, misoprostol and dinoprostone are the most frequently used methods for labor induction [4]. Cervical ripening is a complex process that results in physical softening and distensibility of the cervix, ultimately leading to partial cervical effacement and dilatation [3].

The purpose of the current study was to compare the effectiveness and safety of the pessary to intravaginal dinoprostone gel for induction of labor.

\section{MATERIALS AND METHODS}

Ethical Approval: The Study was approved by the Department of Obstetrics and Gynecology at Security Forces Hospital.

\section{Design and Setting}

The study population was identified from the hospital clinical database and included all women induced at security forces hospital, Riyadh from the duration of January till June 2019

\section{Subjects \\ - Singleton pregnancy \\ - Cephalic presentation \\ - Intact membranes \\ - Gestational age of $\geq \square 37$ weeks \\ - Unscarred Uterus}

Any patient not meeting the above criteria or has a contraindication for normal vaginal delivery was excluded from the study.

Dinoprostone intravaginal gel (Prostin® E2) 1 mg was used for induction of labor and Dinoprostone intravaginal pessaries (Propess) $10 \mathrm{mg}$ were introduced.

The primary outcomes were: incidence of uterine Tachysystole. Uterine Tachysystole was defined as more than five contractions in ten minutes for duration of at least 20 minutes. Secondary outcomes were: caesarean birth and oxytocin augmentation.

Demographic and clinical data were extracted from the hospital clinical database and from the mother's and baby's clinical records. Data on the number of pessaries or gel applications used by each woman in this study were collected.

The protocol for induction of labour at Security forces hospital during the period of the study was as follows: the pessary or gel was inserted into the posterior vaginal fornix if a pre-induction cardiotocograph showed no abnormality. A repeat cardiotocograph was performed 1-hour postinsertion and also when the woman reported regular contractions, ruptured membranes or if there was other concern.
Dinoprostone pessaries were removed if the membranes ruptured spontaneously, when labor was established (painful regular contractions), or at $24 \mathrm{~h}$ postinsertion according to manufacturer's recommendations. If labor had not intervened within 24 $\mathrm{h}$ of initiation of induction vaginal examination was performed to assess whether artificial rupture of membranes was possible or whether a further pessary was needed.

For induction with intravaginal gel, $1 \mathrm{mg}$ was inserted into the posterior fornix with a Bishop score of $\leq \square 6$ were given $1 \mathrm{mg}$ gel with 4 doses maximum, every dose 6 hours apart. Women treated with gel were reexamined at six hourly intervals again according to manufacturer's recommendations to determine whether artificial rupture of the membranes was possible or if further gel insertion was required. Oxytocin infusion was prescribed if labor did not establish after spontaneous or artificial rupture of the membranes or if progress in labor was considered unsatisfactory as determined by the medical team.

\section{STATISTICAL METHODS}

Data were analysed using SPSS Version 11 (IBM, Armonk, NY, USA). Continuous data are presented as mean (standard deviation) if normally distributed or median (interquartile range) if nonparametric, and between-group comparisons were analyzed using Student's $t$-test or Wilcoxon rank sum tests. Frequency data were compared between groups using chisquare and Fisher's exact tests as appropriate. Statistical significance was defined at $P<0.05$.

\section{RESULT}

A total of 200 women were included in the study, of who 100 received dinoprostone pessary and 100 dinoprostone intravaginal gel.

Demographic characteristics, indication for induction and initial Bishop Score did not differ by induction preparation. Mode of birth and need for oxytocin augmentation did not differ by induction preparation for either nullipara or multipara. However, nullipara given the pessary experienced significantly longer median insertion to vaginal birth interval and a smaller proportion delivered within $24 \mathrm{~h}$ of commencing induction (52.5\%) vs (65.4\%), RR 0.80, CI 0.70-0.92).

Tachysystole was diagnosed by the research team in 33 women, $(4.5 \%)$ of those received the pessary and $(2.4 \%)$ of those received gel (Table 4$)$. In terms of time of onset of Tachysystole, one case occurred within one hour of pessary insertion, 13 cases between one and six hours, seven cases between six and $12 \mathrm{~h}$ and a single case occurred after $12 \mathrm{~h}$ after pessary insertion. Fetal heart rate abnormalities associated with Tachysystole were evident in $(2.8 \%)$ women who were induced with 
the pessary, of whom $(1.8 \%)$ were given tocolytics by their attending clinician in the form of Terbutlaine subcutaneously. Caesarean section for suspected fetal compromise associated with uterine Tachysystole was performed in seven women in the pessary group and one in the gel group. There was no correlation between the initial Bishop score and the occurrence of uterine Tachysystole. (4.1\%) women in the pessary group had a postpartum haemorrhage with estimated volume of $\geq$ $1000 \mathrm{~mL}$ compared to $(2.2 \%)$ women in the gel group $(P=0.08)$.

Table-1: Incidence of uterine tachysystole by prostaglandin preparation

\begin{tabular}{|l|c|c|c|}
\hline & Pessary $(\boldsymbol{n}=\mathbf{1 0 0})$ & Gel $(\boldsymbol{n}=\mathbf{1 0 0})$ & RR $(\mathbf{9 5 \%}$ CI) \\
\hline Tachysystole & $4.5(4.5 \%)$ & $2.4(2.4 \%)$ & $1.9(0.9-3.8)$ \\
\hline Tachysystole with abnormal fetal heart rhythm & $2.9(2.9 \%)$ & $0.4(0.4 \%)$ & $6.5(1.5-28.6)$ \\
\hline Tocolytic treatment for Tachysystole & $1.8(1.8 \%)$ & $0.2(0.2 \%)$ & $8.4(1.1-66.1)$ \\
\hline Caesarean performed for Tachysystole & $1.4(1.4 \%)$ & $0.2(0.2 \%)$ & $6.5(0.8-53.0)$ \\
\hline
\end{tabular}

$\mathrm{CI}$, confidence interval; RR, relative risk.I

\section{DISCUSSION}

This study performed comparing birth outcomes in women induced with dinoprostone pessaries compared to dinoprostone intravaginal gel. However, in view of the retrospective nature of the study, the data need to be interpreted with caution. We did not find any substantial benefit from the use of dinoprostone pessaries compared to dinoprostone intravaginal gel for induction of labour.

Nullipara induced with dinoprostone pessaries had higher rates of oxytocin augmentation in labour. Uterine tachysystole, specifically cases associated with abnormal fetal heart rate patterns or requiring tocolytic therapy, appeared to be more common in women who received pessaries.

The longer insertion to birth interval for nulliparous pessary users in our study might be explained by the fact that vaginal examination was not repeated before $24 \mathrm{~h}$ if women did not report regular contractions compared to six hourly examinations which were usual for the gel group.

As prostaglandin works by ripening the cervix, artificial rupture of the membranes could possibly have been performed earlier if vaginal examination had been performed as frequently in women who received pessaries as in those who received dinoprostone gel. While more frequent vaginal examinations to assess readiness for artificial rupture of membranes could be undertaken in women induced with pessaries, this would negate the advantage of reduced numbers of vaginal examinations in pessary users compared to gel users.

We are unsure about the mechanism of this possible increased rate of tachysystole in the pessary group. It is possible that because vaginal examination was performed less often in these women compared to those who received gel that labour was not diagnosed as early and dinoprostone pessaries were left in place after the onset of labour in some women. However, it is also interesting to note that most of cases of tachysystole occurred within six hours of insertion, which suggests that the majority of tachysystole cases might not have been prevented even if vaginal examinations were performed at six hours post- pessary insertion as they were in most women who received gel. In these women, the mechanism is unknown but is less likely to be due to delay in diagnosis of labour. Another notice with the application of the dinoprostone that some of them inserted with K-way jelly or even with saline which may increase the released dose of the dinopreston.

Rath in a recent review about the dinoprostone pessary concluded that the incidence of uterine tachysystole was comparable to other formulations including misoprostol and suggested that dinoprostone pessaries could be utilised in an outpatient setting [7].

Three non-Cochrane meta-analyses [5-7] of randomised trials have studied controlled-release dinoprostone pessaries .The conclusions concluded that

1) Dinoprostone pessaries resulted in fewer vaginal births within $12 \mathrm{~h}$ than other prostaglandin preparations including misoprostol, intracervical and intravaginal gel with no difference in caesarean rate, incidence of tachysystole or need for oxytocin

2) Did not report any difference in mean time to birth, caesarean rate or uterine tachysystole in women who received the pessaries compared to those who received the gel preparation

3) Concluded that the dinoprostone pessary might be as effective as intracervical gel as a cervical ripening agent, but was associated with an increased incidence of excessive uterine activity. These studies were highly heterogeneous as they included studies that compared dinoprostone pessaries with a wide range of induction agents, including misoprostol.

A another Cochrane review [8,9] of vaginal prostaglandin for induction of labour at term concluded that prostaglandin was effective for inducing labour, but more studies were needed to compare different types of 
prostaglandin in terms of their safety and effectiveness and we agree with this view.

\section{CONCLUSIONS}

In this retrospective study, use of dinoprostone vaginal pessaries for induction of labour resulted in no advantage compared with dinoprostone intravaginal gel and clinically significant tachysystole appeared more common. Large randomized controlled trials are necessary to confirm these findings.

\section{Conflict of interest}

The authors state no conflict of interest.

\section{REFERNCES}

1. Report on Maternity. (2006). Maternal and Newborn Information 2003, New Zealand Health Information Service, 34.

2. Rand, L., Robinson, J.N., Economy, K.E., Norwitz, E.R. (2000). Post-term induction of labor revisited. Obstet Gynecol, 96:779-783.

3. Sanchez-Ramos, L., Olivier, F., Delke, I. (2003). Labor induction versus expectant manages- ment for postterm pregnancies: a systematic review with meta-analysis. Obstet Gynecol, 101:1312-1318.

4. Papanikolaou, E.G., Plachouras, N., Drougia, A., Andronikou, S. (2004). Comparison of misoprostol and dinoprostone for elective induc- tion of labour in nulliparous women at full term: a randomized prospective study. Re- prod Biol Endocrinol, 2:70.

5. Tan, T.C., Yan, S.Y., Chua, T.M., Biswas, A., Chong, Y.S. (2010). A randomised controlled trial of low- dose misoprostol and dinoprostone vaginal pessaries for cervical priming. BJOG. 117:1270 1277.

6. Calder, A.A., Loughney, A.D., Weir, C.J., Barberd, J.W.(2008). Induction of labour in nulliparous and multiparous women: a UK, multicentre, open-label study of intravaginal misoprostol in comparison with dinoprostone. BJOG.115:1279-1288.

7. Rath, W. (2005). A clinical evaluation of controlled released dinoprostone for cervical ripening - A review of current evidence in hospital and outpatient settings. J Perinat Med. 33: 491-499.

8. Sanchez-Ramos, L., Kaunitz, A.M., Delke, I., Gaudier, F.L. (1999). Cervical ripening and labor induction with a controlled-release dinoprostone vaginal insert: A meta-analysis. Obstet Gynecol, 94: 878-883

9. Hughes, E.G., Kelly, A.J., Kavanagh, J. (2001). Dinoprostone vaginal insert for cervical ripening and labor induction: A meta-analysis. Obstet Gynecol, 97: 847-855.

10. Crane, J.M., Bennett, K.A. (2000). A meta-analysis of controlled-release prostaglandin for cervical ripening and labour induction. J SOGC. 22: 692 698. 